\title{
Absolute and uniform convergence of spectral expansion in the eigenfunctions of a third-order ordinary differential operator
}

\author{
AYGUN GARAYEVA \\ Department of Computational mathematics, \\ Baku State University \\ Baku, AZERBAIJAN
}

\begin{abstract}
In this paper studied the convergence of spectral expansions of functions of the class $W_{1}(G), G=(0,1)$ in eigenfunctions of an ordinary differential operator of third order with integral coefficients. Sufficient conditions for absolute and uniform convergence are obtained and the rate of uniform convergence of these expansions on the interval $\bar{G}$ is found.
\end{abstract}

Key-Words: - absolute and uniform convergence, eigenfunction, spectral expansion

Received: April 4, 2020. Revised: June 2, 2020. Accepted: June 22, 2020. Published: June 29, 2020.

\section{Introduction}

It is well known that any function in the domain of a self-adjoint differential operator can be expanded in a uniformly convergent series in the eigenfunctions of this operator [1, p.90]. For functions that do not belong to the domain of self-adjoing SturmLiouville operator, the problems of absolute and uniform convergence have been studied in [2-5]. In [2,3] the Sturm-Liouville operator $L u=u+q(x) u$, $x \in G=(0,1)$, with two point self-adjoint boundary conditions (the coefficients in the boundary conditions are real) was considered, and under the condition $\rho$, the absolute and uniform convergence on the interval $\bar{G}$ of the expansions of functions $f(x) \in W_{p}^{1}(G), \quad 1<p \quad 2$, $f(0)=f(1)=0$, in orthonormal eigenfunctions of this operator was proved. The operator $L$ with a real potential $q(x) \in L_{1}(G)$ independent of the specific boundary conditions (in particular, selfadjoint boundary conditions with complex coefficients are also allowed) was considered in [4,5]. The results obtained in [2-5] (for the case of the Sturm-Liouville operator) were generalized in [6] (for the one-dimensional Schrödinger operator).

In the present paper, we study the problems of absolute and uniform convergence of expansions of functions of the class $W_{1}^{\prime}(G)$ in the eigenfunctions of a third-order differential operator [7]. Sufficient conditions for the absolute and uniform convergence of these expansions are obtained, and the rate of uniform convergence is estimated. This study are based on Ilin's spectral method [8].

On the interval $G=(0,1)$, consider the differential operator $L_{u}=u^{(3)}+P_{2}(x) u^{(1)}+P_{3}(x) u$

with coefficients $P_{l}(x) \in L_{1}(G), l=2,3$

By $D(G)$ we denote the class of functions absolutely continuous together with their derivatives of order 2 on the interval $\bar{G}=[0,1]$.

An eigenfunction of operator $L$ corresponding to an eigenvalue is understood as a function $u(x) \in D(G)$ that I s not identically zero and satisfies the equation $L_{u}+{ }_{u}=0$ almost everywhere in $G[8]$.

Let $\left\{u_{k}(x)\right\}_{k=1}$ be a complete orthonormal system in $L_{2}(G)$ consisting of eigenfunctions of the operator $L$, and let $\left\{{ }_{k}\right\}_{k=1}$ be the corresponding system of eigenvalues, $\quad \operatorname{Re}{ }_{k}=0$. By $W_{p}(G), p 1$, we denote the class of functions $f(x)$ absolutely continuous on the interval $\bar{G}$ for which $f^{\prime}(x) \in L_{p}(G)$. We write $\quad{ }_{k}\left(i_{k}\right)^{1 / 3}$, $\operatorname{Im}{ }_{k} 0 ;_{k}=\left(i_{k}\right)^{1 / 3}, \quad$ if $\quad \operatorname{Im} k_{k}<0$, and introduce a partial sum of the spectral expansion of 
the function $f(x) \quad W_{1}(G)$ with respect to the system

$$
\left\{u_{k}(x)\right\}_{k=1}^{\infty}: \delta_{v}(x, f)=\sum_{\mu_{k} \leq v}^{1} f_{k} u_{k}(x), v>0,
$$

where $f_{k}=\left(f, u_{k}\right)=\int_{0}^{1} f(x) \overline{u_{k}(x)} d x$. Study the behavior of the difference $R(x, f)=f(x) \quad(x, f)$.

In this paper, we prove the following results.

Theorem. Assume that a function $f(x) \in W_{1}^{1}(G)$ and a system $\left\{u_{k}(x)\right\}_{k=1}$ satisfy the conditions

$$
\left|f(x) \overline{u_{k}^{(2)}}(x)\right|_{0}^{1} \mid \quad C(f) \quad k\left\|u_{k}\right\|, 0 \quad<2, \quad k \quad 1
$$

and

$$
\sum_{k=2}^{\infty} k^{-1} w_{1}\left(f^{\prime}, k^{-1}\right)<\infty
$$

Then the spectral expansion of the function $f(x)$ with respect to the system $\left\{u_{k}(x)\right\}_{k=1}$ converges absolutely and uniformly on the interval $\bar{G}=[0,1]$, and the estimate

$$
\begin{gathered}
\left\|R_{v}(\cdot, f)\right\|_{C[0,1]} \leq \\
\leq \operatorname{const}\left\{C(f) v^{\alpha-2}+\sum_{k=[v]}^{\infty} k^{-1} w_{1}\left(f^{\prime}, k^{-1}\right)+w_{1}\left(f^{\prime}, v^{-1}\right)+\right. \\
\left.+v^{-1}\left(\left\|f^{\prime}\right\|_{1}+\left(\|f\|_{\infty}+\left\|f^{\prime}\right\|_{1}\right) \sum_{l=2}^{3} v^{2-l}\left\|p_{l}\right\|_{1}\right)\right\}
\end{gathered}
$$

holds, where $\quad{ }_{0}=8, W(x)$ is the modulus of continuity on the space $L_{1}(G),\|\cdot\|_{p}=\|\cdot\|_{L_{p}(G)}$, and the constant is independent of the function $f(x)$.

Corollarys 1 . If the function $f(x) \quad W_{1}(G)$ in theorem satisfies the conditions $f(0)=f(1)=0$, then condition (1) is necessarily satisfied (with the constant $C(f)=0$ ), its spectral expansion with respect to the system $\left\{u_{k}(x)\right\}_{k=1}$ convergence absolutely and uniformly on the interval $\bar{G}=[0,1]$, and the following estimate holds:

$$
\begin{gathered}
\left\|R_{v}(\cdot, f)\right\|_{C[0,1]} \leq \operatorname{cons}\left\{t w_{1}\left(f, v^{-1}\right)+\sum_{k=[v]}^{\infty} k^{-1} w_{1}\left(f^{\prime}, k^{-1}\right)\right. \\
\left.+v^{-1}\left\|f^{\prime}\right\|_{1}\left(1+\sum_{l=2}^{3} v^{2-l}\left\|p_{l}\right\|_{1}\right)\right\}
\end{gathered}
$$

Corollarys 2. If the function $f(x) \quad W_{1}(G)$ in Theorem satisfies the relations $f(0)=f(1)=0$ and $f^{\prime}(x) \in H_{1}^{\beta}(G), 0<1\left(H_{1}(G)\right.$ is the Nikolski class), then conditions (1) and (2) are necessarily satisfied, its spectral expansion converge absolutely and uniformly on the interval $\bar{G}=[0,1]$, and the following estimate holds:

$$
\left\|R_{V}(\cdot, f)\right\|_{C[0,1]} \leq \operatorname{constv}^{-\beta}\left\|f^{\prime}\right\|_{1}^{\beta}, v \geq v_{o}
$$

where $\|f\|_{1}=\|f\|_{1}+\sup _{>0} w_{1}(f$,$) and the$ constant is independent of the function $f(x)$.

Corollarys 3. If the function $f(x) \quad W_{1}(G)$ in Theorem satisfies the relations $f(0)=f(1)=0$ and $w_{1}\left(f^{\prime}, \delta\right)=O\left(\ln ^{-(1+\gamma)} \delta^{-1}\right)$ as $\quad \rightarrow+0$ for $>0$ then conditions (1) and (2) are necessarily satisfied, its spectral expansion converges absolutely and uniformly on $\bar{G}=[0,1]$, and the following estimate holds: $\|R(\square, f)\|_{C[0,1]}=O(\ln \quad)$ as $\rightarrow+\infty$.

\section{Some auxiliary lemmas}

To prove the Theorem, we first prove several assertions. The following lemma was proved in [9,Th.3 ].

Lemma 1. The following estimates hold for the system $\left\{u_{k}(x)\right\}_{k=1}$ and the sequence $\left\{{ }_{k}\right\}_{k=1}$ for any 0 :

$\sum_{0 \leq \mu_{k} \leq \tau}\left\|u_{k}\right\|_{\infty}^{2} \leq$ const $(1+\tau)$ and $\sum_{\tau \leq \mu_{k} \leq \tau+1} 1 \leq$ const . 
Lemma2. (see [7]) The following estimate hold for the system $\left\{u_{k}(x)\right\}_{k=1}$ for any 2 :

$$
\sum_{\mu_{k} \geq \mu} \mu_{k}^{-(1+\theta)}\left\|u_{k}\right\|_{\infty}^{2} \leq C_{1}(\theta), \theta>0,
$$

where $C_{1}()$ is a positive constant.

Lemma 3. Assume that a function $f(x) \quad W_{1}(G)$ satisfies condition (2). Then the estimate

$$
\begin{gathered}
\sum_{\mu_{k} \geq \rho} \mu_{k}^{-1} w_{1}\left(f^{\prime}, \mu_{k}^{-1}\right)\left\|u_{k}\right\|_{\infty}^{2} \leq \\
\leq C_{2}\left\{w_{1}\left(f^{\prime}, \rho^{-1}\right)+\sum_{k=[p]}^{\infty} k^{-1} w_{1}\left(f^{\prime}, \rho^{-1}\right)\right\}
\end{gathered}
$$

holds, where $\quad 8$ and $C_{2}$ is a positive constant independent of and the function $f(x)$.

Proof. Take a positive integer $\mathrm{m}$. By the estimates (4), using the Abel transformation, we obtain the chain of inequalities

$$
\begin{gathered}
\sum_{\rho \leq \mu_{k} \leq[\rho]+m} \mu_{k}^{-1} w_{1}\left(f^{\prime}, \mu_{k}^{-1}\right)\left\|u_{k}\right\|_{\infty}^{2} \leq \\
\sum_{n=[p]}^{[p]+m} n^{-1} w_{1}\left(f^{\prime}, n^{-1}\right)\left(\sum_{n \leq \mu_{k} \leq n+1}\left\|u_{k}\right\|_{\infty}^{2}\right) \leq \\
\sum_{n=[p]}^{[p]+m-1}\left(\sum_{1 \leq \mu_{k} \leq n+1}\left\|u_{k}\right\|_{\infty}^{2}\right)\left\{n^{-1} w_{1}\left(f^{\prime}, n^{-1}\right)-\right. \\
\left.\sum_{1 \leq \mu_{k}<[\rho]+m-1}\left\|u_{k}\right\|_{\infty}^{2}\right)([\rho]+m)^{-1} w{ }_{1}\left(f^{\prime},([\rho]+m)^{-1}\right)+ \\
+\left(\sum_{1 \leq \mu_{k}<[\rho]}\left\|u_{k}\right\|_{\infty}^{2}\right)[\rho]^{-1} w_{1}\left(f^{\prime},[\rho]^{-1}\right) \leq \\
\left.\leq \text { const } \sum_{n=[\rho]}^{[\rho+m-1}(n+1)\left\{f^{\prime},(n+1)^{-1}\right)\right\}+ \\
+ \text { const }([\rho]+m)([\rho]+m)^{-1} w_{1}\left(f^{\prime}, n^{-1}\right)-\left([\rho+1)^{-1} w_{1}\left(f^{\prime},(n+1)^{-1}\right)\right\}+ \\
\leq \text { const }\left\{\sum_{n=[\rho]}^{[\rho \rho+m-1} n^{-1} w_{1}\left(f^{\prime}, n^{-1}\right)+w_{1}\left(f^{\prime},[\rho]^{-1}\right)-w_{1}\left(f^{\prime},([\rho]+m)^{-1}\right)\right\}+ \\
+ \text { const }[\rho][\rho]^{-1} w_{1}\left(f^{\prime},[\rho]^{-1}\right) \leq
\end{gathered}
$$

$$
\begin{gathered}
+ \text { const } w_{1}\left(f^{\prime},([\rho]+m)^{-1}\right)+\text { const } w_{1}\left(f^{\prime},[\rho]^{-1}\right) \leq \\
\leq \text { const }\left\{\sum_{n=[\rho]}^{[\rho]+m-1} n^{-1} w_{1}\left(f^{\prime}, n^{-1}\right)+w_{1}\left(f^{\prime},[\rho]^{-1}\right)+w_{1}\left(f^{\prime},([\rho]+m)^{-1}\right)\right\} .
\end{gathered}
$$

sense the number $\mathrm{m}$ is arbitrary, this together with inequality (2), implies the estimate (6).

Lemma 4. The following estimates hold for the Fourier coefficients $f_{k}$ of a function

$$
\begin{aligned}
& f(x) \in W_{1}^{1}(G) \text { satisfying condition }(1):\left(\begin{array}{cc}
k & 0
\end{array}\right) \\
& \left|f_{k}\right| \leq \text { const }\left\{C(f) \mu_{k}^{\alpha-3}+\mu_{k}^{-1} w_{1}\left(f^{\prime}, \mu_{k}^{-1}\right)+\right. \\
& +\mu_{k}^{-2}\left(\left\|f^{\prime}\right\|_{1}+\left(\|f\|_{\infty}+\left\|f^{\prime}\right\|_{1}\right) \sum_{l=2}^{3} \mu_{k}^{2-l}\left\|P_{l}\right\|_{1}\right\}\left\|u_{k}\right\|_{\infty} .
\end{aligned}
$$

The Lemma 4 was proved un [10,see Lemma 2], in the case $\left\|u_{k}\right\|$ const, $k=1,2, \ldots$ In general case this lemma is proved with similarly way.

\section{Proof of the results}

Prof of theorem. Under the assumptions of theorem, we prove the uniform convergence of the series $\sum_{k=1}^{\infty}\left|f_{k}\right|\left|u_{k}(x)\right|$ on the interval $\bar{G}$. To this end, we represent this series as

$\sum_{k=1}^{\infty}\left|f_{k}\right|\left|u_{k}(x)\right|=\sum_{0 \leq \mu_{k}<v_{0}}\left|f_{k}\right|\left|u_{k}(x)\right|+\sum_{\mu_{k} \geq v_{0}}\left|f_{k}\right|\left|u_{k}(x)\right|$

To estimate the first sum on the right-hands side in (8), we apply the first estimate in (4), $\sum_{0 \leq \mu_{k}<v_{0}}\left|f_{k} \| u_{k}(x)\right| \leq$ const $\|f\|_{1} \sum_{0 \leq \mu_{k} \leq \nu_{0}}\left\|u_{k}\right\|_{\infty}^{2} \leq$ const $\|f\|_{1}$

To estimate the second sum in (8), we use Lemma 4 and the estimates (5) and (6), 


$$
\begin{gathered}
\sum_{\mu_{k} \geq v_{0}}\left|f_{k} \| u_{k}(x)\right| \leq \text { const }\left\{C(f) \sum_{\mu_{k} \geq v_{0}} \mu_{k}^{\alpha-3}\left\|u_{k}\right\|_{\infty}^{2}+\right. \\
+\sum_{\mu_{k} \geq v_{0}} \mu_{k}^{-1} w_{1}\left(f^{\prime}, \mu_{k}^{-1}\right)\left\|u_{k}\right\|_{\infty}^{2}+\left\|f^{\prime}\right\|_{1} \sum_{\mu_{k} \geq v_{0}} \mu_{k}^{-2}\left\|u_{k}\right\|_{\infty}^{2}+ \\
\left.+\left(\|f\|_{\infty}+\left\|f^{\prime}\right\|_{1}\right) \sum_{l=2}^{3}\left(\sum_{\mu_{k} \geq v_{0}} \mu_{k}^{-2}\left\|u_{k}\right\|_{\infty}^{2}\right)\right\} \leq
\end{gathered}
$$$$
\begin{gathered}
\leq \text { const }\left\{C(f) v_{0}^{\alpha-2}+\sum_{n=\left[v_{0}\right]} n^{-1} w_{1}\left(f^{\prime}, n^{-1}\right)+w_{1}\left(f^{\prime},\left[v_{0}\right]^{-1}\right)\right. \\
\left.+\left\|f^{\prime}\right\|_{1}\left[v_{0}\right]^{-1}+\left(\|f\|_{\infty}+\left\|f^{\prime}\right\|_{1}\right) \sum_{l=2}^{3}\left\|P_{l}\right\|_{1}\left[v_{0}\right]^{1-l}\right\}<\infty
\end{gathered}
$$

inequality $\|f\| \quad\|f\|_{1}$, which holds for any function $f(x) \quad W_{1}(G)$ satisfying the relations $f(0)=f(1)=0$ indeed, if $f(0)=f(1)=0$ and $f(x) \quad H_{1}(G)$, then we have $C(f)=0$, and the following chain of inequalities is satisfied $\left({ }_{0}\right)$ : $C(f) v^{\alpha-2}+\sum_{k=[v]}^{\infty} k^{-1} w_{1}\left(f^{\prime}, k^{-1}\right)+w_{1}\left(f^{\prime}, v^{-1}\right)+v^{-1} \times$ )$_{\times}\left(\left\|f^{\prime}\right\|_{1}+\left(\|f\|_{\infty}+\left\|f^{\prime}\right\|_{1}\right) \sum_{l=2}^{3} v^{2-l}\left\|p_{l}\right\|_{1}\right) \leq \sup _{\delta>0}\left(\delta^{-\beta} w_{1}\left(f^{\prime}, \delta\right)\right)$
$\left(\sum_{k=[v]}^{\infty} k^{-(1+\beta)}+v^{-\beta}\right)+v^{-1}\left\|f^{\prime}\right\|_{1}+2 v^{-1}\left\|f^{\prime}\right\|_{1} \sum_{l=2}^{3}\left\|P_{l}\right\|_{l} \leq$

Thus, the series (8) converges uniformly on the interval $\bar{G}$. Therefore, the expansion $\sum_{k=1}^{\infty} f_{k} u_{k}(x)$ converges absolutely and uniformly on this interval. By the completeness of the system $\left\{u_{k}(x)\right\}_{k=1}$ in $L_{2}(G)$ and the absolute continuity of the function $f(x)$, we have the identity $f(x)=\sum_{k=1}^{\infty} f_{k} u_{k}(x), x \quad \bar{G}$

To prove the estimate (3), we use Lemmas 2, 3 and 4.

$$
\begin{gathered}
\left\|R_{v}(\cdot, f)\right\|_{C[0,1]}=\left\|f-\delta_{v}(\cdot, f)\right\|_{C[0,1]}= \\
\left\|\sum_{k=1}^{\infty} f_{k} u_{k}(\cdot)-\sum_{\mu_{k} \leq v} f_{k} u_{k}(\cdot)\right\|_{C[0,1]}= \\
\left\|\sum_{\mu_{k}>v} f_{k} u_{k}(\cdot)\right\|_{C[0,1]} \leq \sum_{\mu_{k} \geq v}\left|f_{k}\right|\left\|u_{k}\right\| \leq \\
\leq \text { const } \sum_{\mu_{k} \geq v}\left\{C(f) \mu_{k}^{\alpha-3}+\mu_{k}^{-1} w_{1}\left(f^{\prime}, \mu_{k}^{-1}\right)+\mu_{k}^{-2}\left\|f^{\prime}\right\|_{1}+\right. \\
\left.+\mu_{k}^{-2}\left(\|f\|_{\infty}+\left\|f^{\prime}\right\|_{1}\right) \sum_{l=2}^{3}\left\|P_{l}\right\|_{1} \mu_{k}^{2-l}\right\}\left\|u_{k}\right\|_{\infty}^{2} \leq \\
\leq \text { const }\left\{C(f) v^{\alpha-2}+\sum_{k=[v]}^{\infty} k^{-1} w_{1}\left(f^{\prime}, k^{-1}\right)+w_{1}\left(f^{\prime}, v^{-1}\right)+\right. \\
\left.+v^{-1}\left(\left\|f^{\prime}\right\|_{1}+\left(\|f\|_{\infty}+\left\|f^{\prime}\right\|_{1}\right) \sum_{l=2}^{3} v^{2-l}\left\|p_{l}\right\|_{1}\right)\right\} .
\end{gathered}
$$

The proof of theorem is complete.

Corollary 2 follows from the definition of norm on the space $H_{1}(G)$ and Theorem with regard to the

$$
\leq \text { const }\left\{\left\|f^{\prime}\right\|_{1}+\sup _{\delta>0}\left(\delta^{-\beta} w_{1}\left(f^{\prime}, \delta\right)\right)\right\}[v]^{-\beta} \leq \operatorname{constv}^{-\beta}\left\|f^{\prime}\right\|_{1}^{\beta} .
$$

To prove corollary 3 , it suffices to note that in this case

$$
\begin{gathered}
\sum_{k=[v]}^{\infty} k^{-1} w_{1}\left(f^{\prime}, k^{-1}\right) \leq \\
\leq C_{1}\left(f^{\prime}\right) \sum_{k=[v]}^{\infty} k^{-1} \ln ^{-(1+\beta)} k=O\left(\ln ^{-\beta} v\right), \beta>0
\end{gathered}
$$

\section{References:}

[1] M. A. Naimark Lineinye differensialnye operatory (Linear Differentiol Operators), 1969, Nauka, Moscow.

[2] N.L. Lazetic On uniform convergence of spectral expansions and their derivatives arising by self - adjeint extensions of a one dimensional Schrödinger operator Publ. Inst. 2001, Moth. Nouv. Ser. Vol. 69 (83), 59-77.

[3] N.L. Lazetic On uniform convergence on closed itervals of spectral expansions and their derivatives for functions from $W_{p}^{1}$ Math.Vestnik, 2004, Vol. 56, 91-104.

[4] V.M. Kurbanov Dependence of rate of equiconvergence on the module of continuity of potential of the Sturm-Liouville operator Stud. Sci. Math. Hung. 2004 Vol.41, No3, 347-364.

[5] V.M. Kurbanov, R.A Safarov On uniform convergence of orthogonal expansions in eigenfunctions of Sturm-Liouville operator 2004, Trans. Acad. Sci. Azerb. Ser. Phys. Vol.24, No1, 161-167.

[6] V.M. Kurbanov, R.A. Safarov Uniform convergence of biorthogonal expansion corresponding to the Schrödinger operator Proc.Inst. Math.Mech. Natl. Acad. Sci. Azerb. 2004, Vol.20, 63-70. 
[7] V.M.Kurbanov, E.B.Akhundova, Absolute and uniform convergence of spectral expansion of the function from the class $W_{p}(G), p>1$ in eigenfunctions of third order differential operator Publ. De l'inst. Mat. 2017 Vol.101 No115, 169-182.

[8] V.A.Il'in Spectral Theory of Differential Operators, 1991, Nauka Moscow

[9] V.M. Kurbanov On the Distribution of eigenvalues distribution and a Bessel property criterion of root functions of a differential operator I Differ. Equations Vol.41, 2005, No4, 489-505.

[10] V.M. Kurbanov, R.I. Shahbazov Absolute convergence of orthogonal expansion in eigenfunctions of odd order differential operator Azer. Jour. of Math. 2018, Vol.8 No2, 152-162.

\section{Creative Commons Attribution}

\section{License 4.0 (Attribution 4.0} International , CC BY 4.0)

This article is published under the terms of the Creative Commons Attribution License 4.0

https://creativecommons.org/licenses/by/4.0/deed.en $\underline{\mathrm{US}}$ 